\title{
Advanced LIGO: non-Gaussian beams
}

\author{
Erika D'Ambrosio ${ }^{1}$, Richard O'Shaugnessy ${ }^{1}$, Kip Thorne ${ }^{1}$, \\ Phil Willems ${ }^{1}$, Sergey Strigin ${ }^{2}$ and Sergey Vyatchanin ${ }^{2}$ \\ ${ }^{1}$ California Institute of Technology, Pasadena, CA, USA \\ ${ }^{2}$ Moscow State University, Moscow, Russia
}

Received 21 August 2003

Published 11 February 2004

Online at stacks.iop.org/CQG/21/S867 (DOI: 10.1088/0264-9381/21/5/074)

\begin{abstract}
By using non-Gaussian, flat-topped beams in the advanced gravitational wave interferometers currently being designed, one can reduce the impact on the interferometer sensitivity of a variety of fundamental disturbances (thermoelastic noise, noise in mirror coatings, thermal lensing, etc). This may make beating the standard quantum limit an achievable goal.
\end{abstract}

PACS numbers: $04.80 . \mathrm{Nn}, 05.40 .+\mathrm{j}, 03.40 . \mathrm{Kf}$

(Some figures in this article are in colour only in the electronic version)

The future generation of gravitational wave interferometers has the ambitious goal of improving the sensitivity of the detectors up to the standard quantum limit and beyond. For this purpose stricter requirements need to be met by all the detector subsystems. The sensitivity must be dominated by sources of fundamental noise; all technical noises must not contribute more than those. By technical noise we mean any non-fundamental disturbance that is related to the performance of some subsystem, such as fluctuations in the laser frequency or in the input power. Such noises depend on the quality of the equipment and some of them will be counteracted by control systems. Fundamental noises are the direct result of physical mechanisms such as the Brownian motion caused by thermal activity at non-zero temperature, or the statistical errors that affect the measurement due to discreteness of photons.

In the frequency range of maximum sensitivity, thermal noise is the dominant fundamental noise source. There are several kinds of contributions to the thermal noise; the reflection of the beam from the surface of the mirror is affected by any random motion of the surface with respect to the centre of mass.

The thermal contact of any system with a heat bath keeps the system in thermal equilibrium. When the system is independent of the bath, energy is continuously transmitted between them, and the resulting thermal fluctuations are governed by the fluctuationdissipation mechanism.

For solid bodies such as an interferometer mirror, the mode-mode couplings associated with internal dissipation are the main sources of thermal fluctuations; in this case all the other degrees of freedom serve as a reservoir for each degree of freedom. They exchange energy on some typical time scale $\tau$ and on that same time scale the temperature fluctuates. 


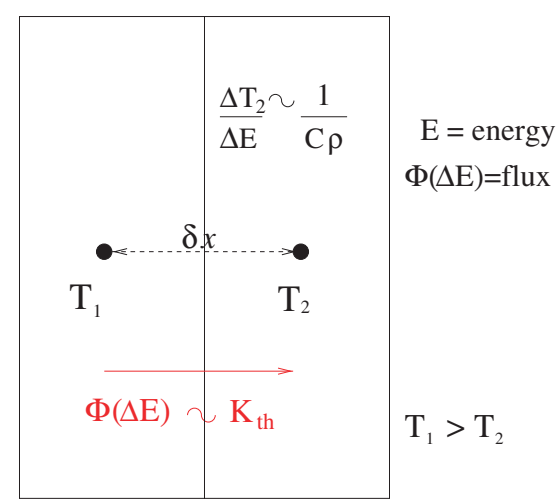

Figure 1. Energy flows between two elements of volume because of $T_{1} \neq T_{2}$. For small $\delta x$ the equality $T_{1}=T_{2}$ is rapidly restored by the transferred heat.

The connection between the random flow of thermal energy among the degrees of freedom and the temperature fluctuations depends on the specific heat $C$ of the material. If $C$ is low, the temperature is sensitive to the circulation of energy and there are substantial local temperature fluctuations around the mean. The length scale $\lambda_{T}$ of these fluctuations depends on the ratio of the thermal conductivity over the specific heat. In figure 1 we consider two adjacent elements of material. In the limit as their centres' separation $\delta x$ goes to zero, they must be at the same temperature. As we increase $\delta x \neq 0$, their instantaneous temperatures will typically be different, $T_{1} \neq T_{2}$, and a diffusive flux of energy will flow from one to the other, proportional to the thermal conductivity $K_{\mathrm{th}}$. This flux can quickly restore $T_{1}=T_{2}$ if the specific heat is small so that the temperature response is immediate. The result is a diffusion constant $\sim K_{\text {th }} / C$ for the heat. From these arguments we can deduce that

$$
\lambda_{T} \propto \sqrt{\frac{K_{\mathrm{th}}}{C} \tau}
$$

is the typical length scale over which the temperature can be made homogeneous by diffusive heat flow during a time $\sim \tau$ and fluctuation-dissipation considerations imply that this is also the length scale for temperature fluctuations on that time scale.

We are interested in fluctuations happening on the frequency scale

$$
\tau^{-1}=f_{g w},
$$

since they can affect the detection of gravitational-wave signals at $f_{g w}$. The characteristic area over which the temperature fluctuates on this time scale is of the order of

$$
\lambda_{T}^{2} \simeq \frac{K_{\mathrm{th}}}{C \rho} \tau=(3.3 \mathrm{~mm})^{2} \frac{\tau}{\mathrm{s}}
$$

for the following parameters:

$$
K_{\text {th }}=33.0 \mathrm{~W} \mathrm{~m}^{-1} \mathrm{~K}^{-1}, \quad C=770.0 \mathrm{~J} \mathrm{~kg}^{-1} \mathrm{~K}^{-1}, \quad \rho=3980.0 \mathrm{~kg} \mathrm{~m}^{-3}
$$

typical of the sapphire substrates planned for advanced LIGO's mirrors.

Each region with a momentarily different temperature $T$ is subject to a different thermal expansion, which varies on the same time scale $\tau$ as its $T$. Therefore, the mirror surface will be spoiled by fluctuating irregularities at frequency $f_{g w}=\tau^{-1}$, which are coherent over areas as large as $\lambda_{T}^{2}$. These fluctations are called thermoelastic noise, and their importance for LIGO was pointed out by Braginsky et al [1]. The probe we use in Advanced LIGO 


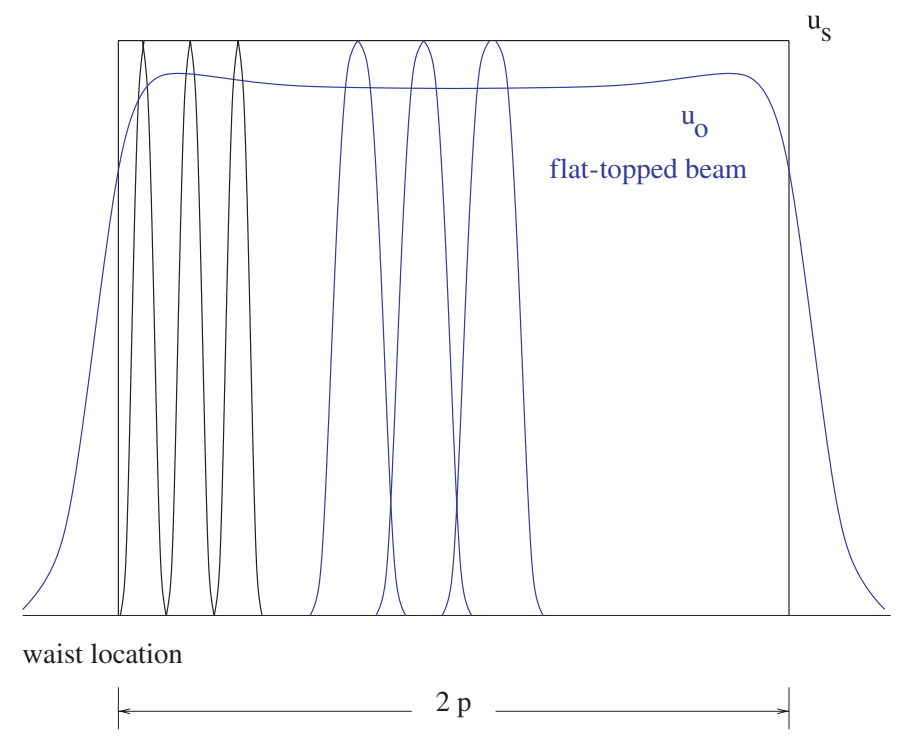

Figure 2. We can imagine building a flat-topped ('mesa') beam for Advanced LIGO, mathematically, by specifying its form at the waist (half way between the two mirrors), where the phase front is planar. We specify that form as a superposition of Gaussian functions with their centres uniformly distributed over a circle of diameter $2 p$ [2]. As each Gaussian propagates down the tube, its width broadens and phase front bends. The resulting phase front for the total field is evaluated at the mirror location and the surface of the mirror is designed so that it exactly matches that phase front. The resulting mirror shape resembles a Mexican hat (sombrero), so we call it a Mexican hat $(\mathrm{MH})$ mirror [2]. A resonator with such a mirror at each end will have the mesa beam as its optical mode. If the cavity is driven by a different light field, only that portion which overlaps with the mesa beam can build up power inside the resonator. An input beam that is as closely matched with the mesa beam as possible should be used. The whole interferometer should be designed accordingly, with the signal recycling mirror matching the phase front of the mesa beam exiting the Fabry-Perot.

for measuring the position of the mirror is a Gaussian-shaped laser beam with a spot size $w \sim 6 \mathrm{~cm}$. Averaging the displacement of the thermoelastically fluctuating surface by the beam's Gaussian distribution of light power is not optimal; a better averaging can be achieved with a flat light profile. How flat the light intensity can be is a trade-off between the size of the mirrors and diffraction.

Diffraction prevents us from creating in LIGO a beam with a rectangular power profile as in figure 2. We can imagine that the rectangular shape is due to an overlap of infinite delta functions that we express as Gaussian functions, having infinitely small width. The diffraction angle for each Gaussian would be very large because of its steep edge, causing the rectangular beam to spread enormously as it propagates. This spreading can be reduced by making the edge of the profile smoother; this can be achieved by making the widths of the Gaussian functions larger. However, if they become too large, the shape of their superposed beam will be too spread out. Therefore, there is an optimal width for the Gaussians for which the superposed intensity is flat in the centre and falls off at the edges at a rate that leads only to modest spreading when travelling down a LIGO arm cavity. This optimal width only depends on the cavity's length and laser wavelength. The permissible width of the resulting 'flat-topped' beam is determined by how much light we allow to be lost off the edges of the 
mirrors. Because the resulting beam has a shape (figure 2) rather like that of the mesas that occur in the deserts of the Southwestern United States, we call it a mesa beam.

Besides the width of the Gaussian functions, the mesa beam is characterized by the extension of their overlap over a disc of diameter $2 p$. Requiring a limit for the diffraction loss due to the finite size of the mirrors fixes $p$. More accurate studies are needed to explore possible alternatives; we are currently addressing the optimization problem.

The influence of each arm-cavity mirror on the interferometer is proportional to

$$
\delta h(t)=\iint_{x^{2}+y^{2} \leqslant m^{2}}|u(x, y)|^{2} \delta h(x, y, t) \mathrm{d} x \mathrm{~d} y,
$$

where $\delta h(x, y, t)$ is the mirror height at transverse location $x, y$ and time $t,|u(x, y)|^{2}$ is a unit-norm function proportional to the beam's light power distribution, and $2 m$ is the diameter of the mirror. Three of us $[3,2]$ have computed the influence of the mirror's thermoelastic noise on the power spectral density $S_{h}(f)$ of this $h(t)$. They find that by switching, in advanced LIGO, from a Gaussian beam to a mesa beam, with beam widths adjusted for the same diffraction losses of about $10 \mathrm{ppm}$ per bounce, $S_{h}$ can be reduced by a factor $\simeq 3$ at all relevant frequencies.

There are other disturbances, in addition to thermoelastic noise, that may benefit from a flat, mesa-like intensity profile for the beam. Two examples are coating mechanical losses and thermal lensing. We shall discuss thermal lensing briefly:

Because of the high gain of the Fabry-Perot cavities, the power hitting the mirrors is so large as to deposit enough heat on each bounce to change the mirror's refractive index. In sapphire mirrors, but not fused silica, the thermal expansion due to deposited heat is also significant. Both effects produce thermal lensing which distorts the light beam. To counteract the temperature gradients that produce this thermal lensing in both transmission and reflection, the inhomogeneity of the absorbed power must be compensated by heating the cold portions. Direct measurements of thermal lensing have been made in LIGO I. There is a quantitative disagreement between the measurements and the predicted magnitude of the effect, according to the model and the parameters that have been used to calculate the mirror curvatures for the full laser power load; such disagreement is currently under investigation.

If sapphire substrates are selected for the mirrors of Advanced LIGO, the thermal lensing is expected to be large enough to influence the fundamental mode $u(x, y)$ of the arm cavities. Correspondingly, to make that mode have a mesa-beam shape when the cavities have been brought up to full power, it will be necessary to take account of the thermal lensing when designing the mirror shapes. The task of designing those shapes requires nonlinear modelling, and there are several methods to converge to a stable solution [4].

A variety of objections has been raised against using non-spherical mirrors in optical resonators. Some of these objections can only be evaluated reliably by experimental tests, and for this reason a table top experiment is being planned [5]. The main motivation for this experiment is to check whether the resonating mode has the features we expect.

We have carried out extensive analyses of the response of a mesa beam to errors in the mirrors' positions, tilts and figure shapes, in a full size LIGO gravitational wave antenna $[9,3,2]$. We find that the interferometer's shot noise is more sensitive, by a factor of a few, to arm-cavity mirror errors when mesa beams are used than for the baseline Gaussian design. Our numerical results generally have an intuitive interpretation. For example, the magnitude of tilt of an arm cavity mirror that increases the level of shot noise by $1 \%$ is $\sim 0.02 \mu \mathrm{rad}$. The corresponding tilt for the baseline, Gaussian-beam configuration is five times larger. This result is about what we would expect from examining the ratio between the diffraction angle of the baseline Gaussian beam for Advanced LIGO and the mesa beam. Such a ratio is $\sim 6$, 


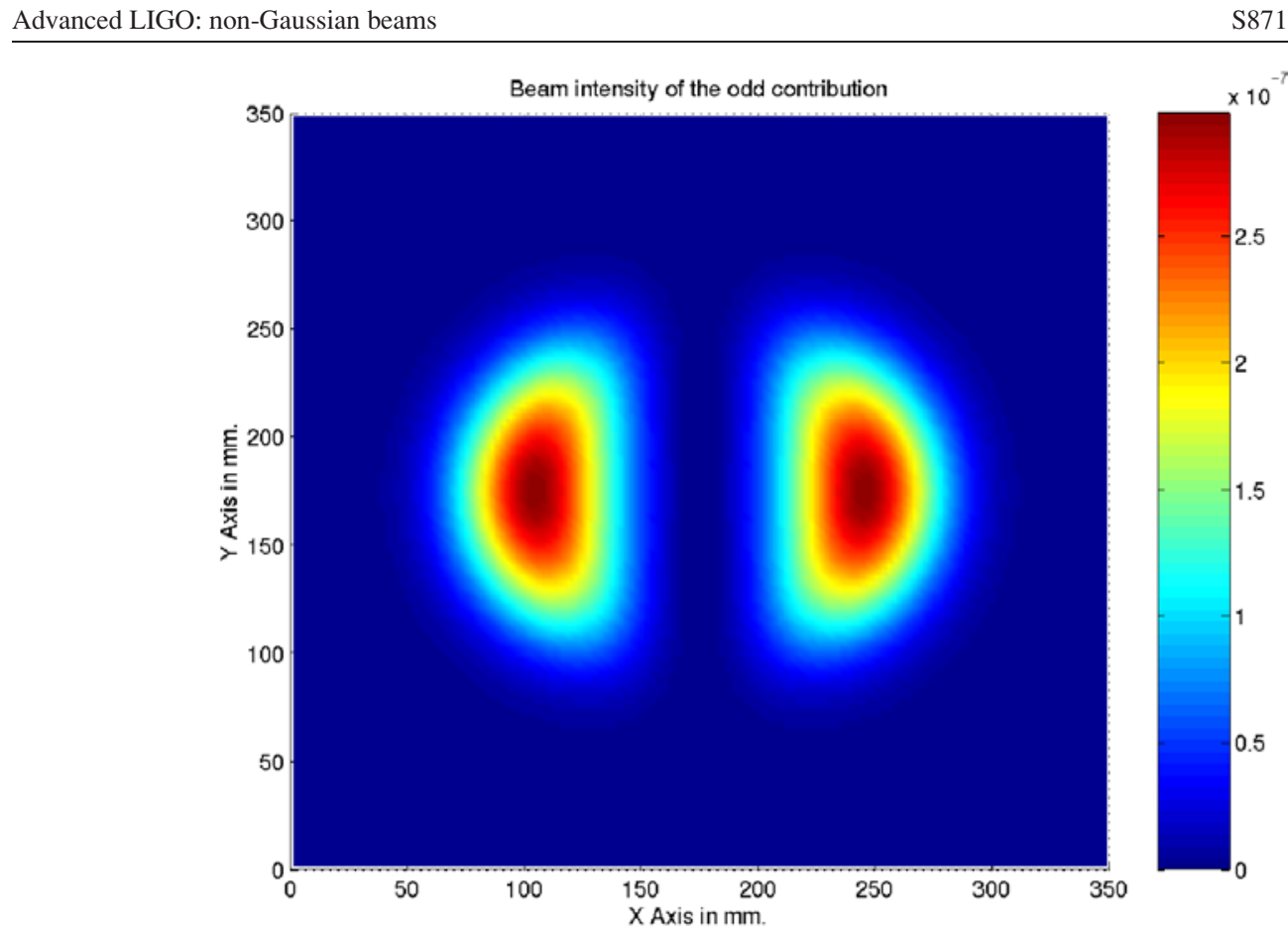

Figure 3. When a mirror in one arm cavity is tilted, the beams reflected from the perturbed and the unperturbed arm are no longer the same. The optical mode excited by the mirror tilt exits the dark port of the beamsplitter. Its intensity distribution, for the case of mesa beams, is shown here; the excited component is similar to the TEM10 mode corresponding to the baseline Gaussian beams.

which means the Gaussian beam can tolerate an angle error about six times larger than the mesa beam and still be focused ${ }^{3}$.

The fact that the response of a mesa beam to errors in the mirror tilts and positions is analogous to the response of a Gaussian beam is of great importance, thereby permitting the same techniques as in the Gaussian case to be used for monitoring and controlling the mirror tilt and position [7, 8]. As an example, the tilt of a mirror mixes into the arm cavity's mesa beam an antisymmetric arm-cavity mode whose intensity distribution is shown in figure 3 . This admixed mode closely resembles the TEM10 Hermite-Gaussian mode that is admixed when the unperturbed beam is Gaussian. In figure 4 we illustrate how this admixture can be used to control the mirror alignment both in an interferometer with a mesa beam and in one with a Gaussian beam. The carrier field resonating inside the perturbed cavity propagates along a different axis than in the unperturbed cavity and therefore its intensity (superposition of original mode and admixed mode) is off centre, giving rise to a feedback signal that corrects the tilt $[7,8]$. One of us has tested the principles of alignment control in a series of numerical simulations aimed at characterizing the response of the mesa beam to mirror tilt and surface imperfections [9].

We have also analysed the effects of mirror figure errors on an Advanced LIGO interferometer with mirrors supporting mesa beams. We assumed shapes for the figure errors based on the measured shape errors of a LIGO-I beam splitter, with the measured data manipulated in several ways to set requirements on the figure error of the mirror profile

\footnotetext{
3 For a definition of the diffraction angle see for example [6].
} 


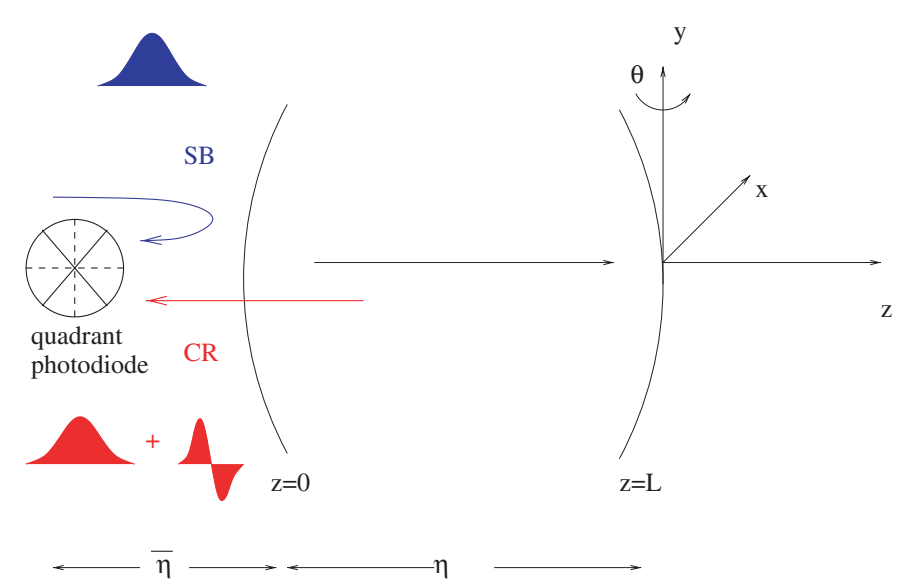

Figure 4. When a mirror is tilted the optical axis of its cavity is affected and therefore the centre of the intensity distribution is shifted, providing a signal that can be used to control the mirror tilt.

[2]. We have expressed the results of our analyses in terms of the increased shot noise as a function of the peak-to-valley figure error, $\operatorname{Max}[\Delta z][9,3,2]$.

To keep the shot noise increase below $1 \%$ when all four arm-cavity mirrors are affected by equal magnitude but uncorrelated distortions, one must impose the constraints

$$
\begin{array}{ll}
\operatorname{Max}[\Delta z] \simeq 100 \mathrm{~nm} & \text { in the outer area } \quad r \geqslant 12.2 \mathrm{~cm} \\
\operatorname{Max}[\Delta z] \simeq 5 \mathrm{~nm} \quad \text { in the central area } \quad r \leqslant 9.6 \mathrm{~cm} .
\end{array}
$$

Not surprisingly there is a much higher tolerance to deviations from the ideal figure in the outer region than in the inner.

Our modelling shows that tilts and figure errors for the MH mirrors that bound the Advanced-LIGO signal recycling (SR) cavity have an approximately three times larger influence on shot noise than tilts and figure errors for the arm cavity MH mirrors, and correspondingly the constraints on the SR cavity mirrors are approximately three times more severe [2]. For the baseline Gaussian design, the SR mirror constraints are only about 1.5 less serious than for mesa beams. For both cases it may be desirable to make the SR cavity substantially less degenerate than currently planned, so as to reduce the sensitivity to SR mirror errors.

In our analyses, we found that a small lateral displacement of a $\mathrm{MH}$ mirror does not much influence the mesa beam in the mirror's cavity, since the mirror's central area is quite flat and the difference between the unperturbed and perturbed surfaces is concentrated along the external area [10,2].

In conclusion, our analyses suggest that the practical challenges that the use of nonspherical mirrors would entail are within reach of the state-of-the-art technology. However, further modelling and experimental work is needed, especially on the reproducibility with which our proposed mirror profiles can be manufactured. ${ }^{4}$

4 Jean Marie Mackowski believes the desired MH figure can be produced by coating, with an error of about two nanometres. This accuracy is better than the requirement we have set on the peak-to-valley deviation in the central area of the arm-cavity mirrors, but is comparable to that required for the SR-cavity mirrors with the currently planned SR-cavity degeneracy; hence our exploration of making this cavity less degenerate. 


\section{Acknowledgments}

For helpful discussion and advice we especially thank Stan Whitcomb. We have also benefitted from discussions with colleagues in the LIGO Scientific Collaboration and elsewhere, among them: Raymond Beausoleil, Alessandra Buonarmo, Vladimir Braginsky, Yanbei Chen, Peter Fritschel, Gregg Harry, Bill Kells, David Shoemaker, Robert Spero, Ken Strain, Rai Weiss, Farid Khalili and Mike Zucker. We are particularly grateful to Garilynn Billingsley. This work was supported by the National Science Foundation under the grants PHY-0098751, PHY-0099568 and PHY-9210038 and by the Russian Foundation under \#96-02-16319a and \#97-02-0421g. We also acknowledge the Center for Advanced Computing Research of Caltech for providing the supercomputer facilities. We are grateful to Larry Wallace and the system administration group he leads.

\section{References}

[1] Braginsky V B, Gorodetskay M L and Vyatchanin S P 1999 Thermodynamical fluctuations and photo-thermal shot noise in gravitational wave antennae Phys. Lett. A 2641

[2] D'Ambrosio E, O'Shaughnessy R, Thorne K S, Strigin S and Vyatchanin S 2003 Reducing thermoelastic noise in gravitational wave interferometers by flattening the light beams Phys. Rev. D submitted

[3] O'Shaughnessy R, Strigin S and Vyatchanin S 2003 The implications of Mexican-hat mirrors: calculations of thermoelastic noise and interferometer sensitivity to perturbation for the Mexican-hat-mirror proposal for advanced LIGO Phys. Rev. D submitted

[4] Beausoleil R, Gustafson E, Fejer M, D’Ambrosio E, Kells B and Camp J 2003 Model of thermal wave-front distortion in interferometric gravitational-wave detectors: I. Thermal focusing J. Opt. Soc. Am. B 206

[5] Willems P and D'Ambrosio E 2003 Proposal for a tabletop mexican hat beam experiment LIGO Technical Note Publically available from the LIGO Document Control Center (DCC)

[6] Kogelnik H and Li T 1966 Appl. Opt. 5

[7] Sigg D 1996 Principles of calculating alignment signals in complex resonant optical interferometers LIGO$P 960024-A-D$ Available from the DCC

[8] Anderson D Z 1990 Alignment of resonant optical cavities Appl. Opt. 29 394-403

[9] D'Ambrosio E 2003 Non-spherical mirrors to reduce thermoelastic noise in advanced gravitational wave interferometers Phys. Rev. D 67 ID 102004

[10] D'Ambrosio E 2003 Geometrical cryogeny, Viewgraphs are available from the LIGO DCC 